\title{
PERCEPCIÓN Y EXPECTATIVA DE LOS CIUDADANOS PERUANOS FRENTE AL GOBIERNO ELECTRÓNICO
}

\author{
Diego Cardona, Ph.D. \\ ESADE-UNIVERSIDAD RAMÓN LLULL, BARCELONA, ESPAÑA \\ BECARIO CÁTEDRA UNESCO \\ GERENTE POR PARTE DEL BANCO MUNDIAL DEL PROYECTO SNIES EN COLOMBIA \\ diego.cardona@esade.edu
}

\begin{abstract}
Resumen
Presenta cómo los ciudadanos peruanos evalúan los esfuerzos gubernamentales por implementar iniciativas de gobierno electrónico. Primero se describen los conceptos básicos usados en el artículo, luego se expone brevemente la política peruana de gobierno electrónico y se termina midiendo cómo perciben los ciudadanos el gobierno electrónico y qué esperan de éste, mediante un modelo desarrollado por el autor durante su investigación doctoral (Cardona, 2004). El modelo incluye cinco constructos latentes e independientes: actitud, aptitud, confianza, relevancia y satisfacción, que afectan dos constructos latentes y dependientes: la percepción y la expectativa ciudadanas frente a su interacción con la administración pública a través del uso de las tecnologías de información y comunicación, bajo el efecto de variables ilustrativas relacionadas con los ciudadanos, las instituciones y el entorno.
\end{abstract}

E la actualidad, el gobierno peruano está desarrollando políticas para conducir el país hacia la sociedad del conocimiento, tales como la implementación de iniciativas en el ámbito del gobierno electrónico. Con el propósito de evaluar estas políticas, se han identificado las variables necesarias para medir cómo perciben los ciudadanos el gobierno electrónico y qué esperan de éste. Aunque el trabajo se realizó de manera comparativa para Colombia y el Perú, en este artículo sólo se presentan los resultados del caso peruano.

\section{Definiciones}

Es necesario aclarar que el término sociedad del conocimiento se emplea en este documento para referirse al uso creciente de las tecnologías de información y de comunicación (TIC) y al impacto social, político, cultural y económico que éste causa en la sociedad, los gobiernos y la economía. La sociedad actual está cada vez más interconectada, funciona de manera interactiva, instantánea, es rica en información, informal e incierta (Gualtie- 
ri, 1999). La última de las características que muestra se denomina brecha digital, entendida como las diferencias, entre individuos, hogares, compañías, organizaciones y áreas geográficas, debidas a la oportunidad de acceder a las TIC y al uso que se le da a Internet en las actividades diarias (OECD, 2000). Por su parte, las TIC comprenden el conjunto de actividades que facilitan por medios electrónicos el archivo, procesamiento, transmisión y despliegue interactivo de información (OECD, 2000).

Como núcleo de este artículo, el gobierno electrónico presenta al menos las siguientes características (Cardona, 2004):

- Se relaciona con la aplicación de las TIC.

- Innova las relaciones internas y externas del gobierno con otras agencias gubernamentales, sus propios empleados, las empresas y los ciudadanos.

- Afecta la organización y función del gobierno respecto al acceso a la información, servicios, trámites y participación ciudadana.

- Busca la optimización del uso de los recursos para la obtención de los objetivos gubernamentales.

- Su implementación implica el paso por una serie de fases que no son necesariamente consecutivas.

- $\quad$ Es un medio, no un fin en sí mismo.

\section{La política peruana de gobierno electrónico}

De acuerdo con varios estudios de preparación para el gobierno electrónico, se puede concluir que el Perú representa un caso estándar en el entorno latinoamericano. Por ejemplo, según The Global Information Technology Readiness Report for 2004-2005 (Dutta y Lopez-Claros, 2005), el índice peruano de desarrollo de infraestructura fue de 4,88 puntos en una escala de 1 a 7, puntaje que lo coloca en el lugar 47 entre un total de 75 países analizados. Igualmente, un trabajo de Telefónica del Perú (Bustamante, 2002) encontró que el país presentaba en el 2002 una densidad estimada de computadores personales de 4,79 por cada 100 habitantes, cifra que lo posicionaba en el octavo lugar entre 18 países latinoamericanos. En claro contraste, la densidad de computadoras personales en Estados Unidos era de 62,25.

Respecto a la infraestructura de comunicaciones, la tasa de crecimiento de la década del 90 fue muy elevada, pero insuficiente para cubrir las necesidades del país. A mediados del 2003 , la penetración de la telefonía fija en Lima era de 13 líneas por cada 100 habitantes, mientras que en el resto del país este indicador era de 3,17 por cada 100 habitantes, de modo que la penetración total para el país era de 7,75. Sin embargo, el gobierno peruano ofrece acceso a Internet desde 1991, y en el 2003 había más de 3 millones de usuarios de Internet, número que a mediados del 2004 superaba los 4 millones, con una penetración de usuarios de Internet de $15,4 \%$.

Finalmente, en el campo de lo social la educación está considerada como la clave para crear, adaptar y desplegar conocimiento, pero el sistema educativo peruano no ha impulsado el uso de las TIC como herramienta de aprendizaje ni como elemento crítico. Todavía hoy no existe un documento actualizado de política de 
gobierno electrónico (Pasco, 2005; Bermúdez, 2004; SNI, 2002) ${ }^{1}$. La responsabilidad de actualizar la estrategia nacional de gobierno electrónico fue asignada a la Secretaría de la Presidencia del Consejo de Ministros, a través de la Oficina Nacional de Gobierno Electrónico, Ongei (Parra, 2004).

De acuerdo con el secretario general de la PCM, en el ámbito del gobierno electrónico el país persigue cuatro propósitos:

a) Modernizar y descentralizar la administración pública usando las TIC.

b) Promover el incremento de la productividad a partir del uso intensivo de las TIC.

c) Fomentar el acceso universal al conocimiento y a la información con base en el uso masivo de Internet.

d) Propiciar la sociedad de la información.

\section{La percepción y la expectativa ciudadanas}

\subsection{Fundamento teórico}

A continuación se explica el modelo propuesto para medir la percepción y la expectativa de los ciudadanos frente al gobierno electrónico ${ }^{2}$. La idea general es que la percepción y la expectativa están relacionadas con la actitud, entendida ésta como la predisposición aprendida para actuar hacia un objeto, persona o situa-

1. Conviene aclarar que ya se conocen los borradores del documento de política y es factible que éste se haga público en el 2005.

2. Este modelo es resultado de la tesis doctoral del autor: Las tecnologías de la información y las comunicaciones (TIC) en la relación admi- ción. La actitud incluye dimensiones cognitivas, afectivas, evolutivas y conductuales organizadas de manera sistemática con elementos interrelacionados, de tal manera que un cambio en uno de ellos afecta a los demás (Fishbein y Ajzen, 1980; Rubio, 2000; Henerson, Morris, y FitzGibbon, 1987). Esta aproximación se denomina Teoría de la Acción Razonada (TRA), la cual sostiene que con el objeto de predecir y entender los comportamientos es necesario medir las actitudes hacia éstos. Con este fin se identifican las normas subjetivas y pesos relativos de las actitudes para relacionarlos con los comportamientos siguiendo factores específicos.

La Teoría de la Acción Razonada ha sido la base para otros trabajos sobre este mismo aspecto, como el Modelo de Aceptación Tecnológica (TAM) desarrollado por Davis (Davis, Bagozzi, y Warshaw, 1989; Davis y Venakatesh, 2000; Davis, 1989; Davis y Venakatesh, 1996; Davis y Venkatesh, 2004), la Teoría del Comportamiento Planeado, TPB (Mathieson, 1991), la Teoría de la Descomposición del Comportamiento Planeado, DTPB (Taylor y Todd, 1995), la Teoría de la Riqueza de Información y Presencia Social, SPIR (Gefen y Straub, 1997), la Teoría Cognitiva Social, SCT (Compeau, Higgins y Huff, 1999) y la Teoría de la Difusión de Innovaciones, DOI (Rogers, 1995). Una excelente compilación sobre el tema se puede consultar en The Institute for Advanced Management Systems

nistración pública-ciudadano: la declaración electrónica de impuestos en una evaluación comparativa del caso colombiano y peruano, sustentada en ESADE-Universidad Ramón Llull (Barcelona, España) para optar el grado de Ph.D. in Management Sciences. 
Research (IAMSR), en Finlandia (Han, 2003).

\subsection{Hipótesis}

Como se describe en el documento base de este artículo (Cardona, 2004), las hipótesis de investigación verificadas partieron del hecho de que en el estudio de las TIC en la relación Administración Pública-Ciudadano se observan los constructos independientes Aptitud, Actitud, Satisfacción, Confianza y Relevancia y los constructos dependientes Percepción y Expectativas.

Desde la perspectiva de la percepción, se planteó verificar que en cuanto a la administración pública existe una mayor confianza en los servicios electrónicos que en los servicios prestados a través de medios tradicionales, porque se considera que la información transmitida permanece confidencial, no se viola la privacidad del ciudadano, el proceso ejecutado es seguro, el resultado obtenido es equivalente al obtenido con medios tradicionales y el proceso ejecutado es equivalente al ejecutado con medios tradicionales. Igualmente, se planteó verificar que los servicios electrónicos implementados por la administración pública son relevantes, porque se considera que el proceso ejecutado tiene un responsable claramente identificable, se incrementa la credibilidad de la administración pública, hay mayor facilidad para ejecutar los procesos, se fomenta la innovación en la administración pública y la integración entre sus instituciones. Finalmente, en esta perspectiva de la percepción se planteó verificar que existe una mayor satisfacción con los servicios electrónicos que con los servicios prestados a través de medios tradicionales, porque se considera que los servicios ofrecidos vía Internet son excelentes, la vía electrónica reduce el tiempo y el costo de los trámites, se incrementa la transparencia de la administración pública, se puede acceder a más información que con medios tradicionales y hay disponibilidad de uso las 24 horas de los 7 días de la semana.

Desde la perspectiva de la expectati$v a$, se planteó verificar que: a) el sistema de interacción con la administración pública más deseado es el que se realiza a través de medios electrónicos, b) el proceso más deseado es la realización de trámites, c) el área de mayor interés es el empleo y d) el beneficio más esperado por los ciudadanos es la reducción de costos.

\subsection{Modelo}

Como resultado de la revisión de literatura y aplicando un análisis multivariante para confirmar los resultados, se concluyó que la percepción y la expectativa pueden calcularse como una regresión lineal -con alto nivel de ajuste- de las variables: aptitud, actitud, confianza, relevancia y satisfacción, con unos coeficientes como los que se observan en las ecuaciones siguientes:

$$
\begin{aligned}
\text { Per_Relacion }= & 3,376-0,447 * \mathrm{Ap}+0,229 * \mathrm{Ac}+0,041 * \mathrm{C}+0,227 * \mathrm{R}-0,024 * \mathrm{~S} \\
\operatorname{Exp} \_ \text {Relacion }= & 3,508+0,048 * \mathrm{Ap}+0,232 * \mathrm{Ac}+0,051 * \mathrm{C}+0,120 * \mathrm{R}-0,062 * \mathrm{~S} \\
& \text { Per_Relacion }=-1,185+1,048 * \text { Exp_Relacion }
\end{aligned}
$$

Donde: 
Per_Relacion Nivel de acuerdo con la frase: Considero que los servicios ofrecidos por la administración pública vía Internet han mejorado mi relación con la administración pública.

Exp_Relacion Nivel de acuerdo con la frase: En caso de interacción con la administración pública vía Internet, espero una excelente relación.

Ap Aptitud: habilidades y capacidades de los ciudadanos para usar las TIC en su relación con la administración pública.

Ac Actitud: disposición de los ciudadanos para usar las TIC en su relación con la administración pública.

C Confianza.

R Relevancia.

S Satisfacción.

Una mayor especificación y detalle del modelo se puede consultar en http:// dsi.esade.edu/dcardona/tesis (20 de diciembre, 2004). La representación gráfi- ca de las ecuaciones con sus variables y coeficientes de regresión se puede observar en la figura adjunta.

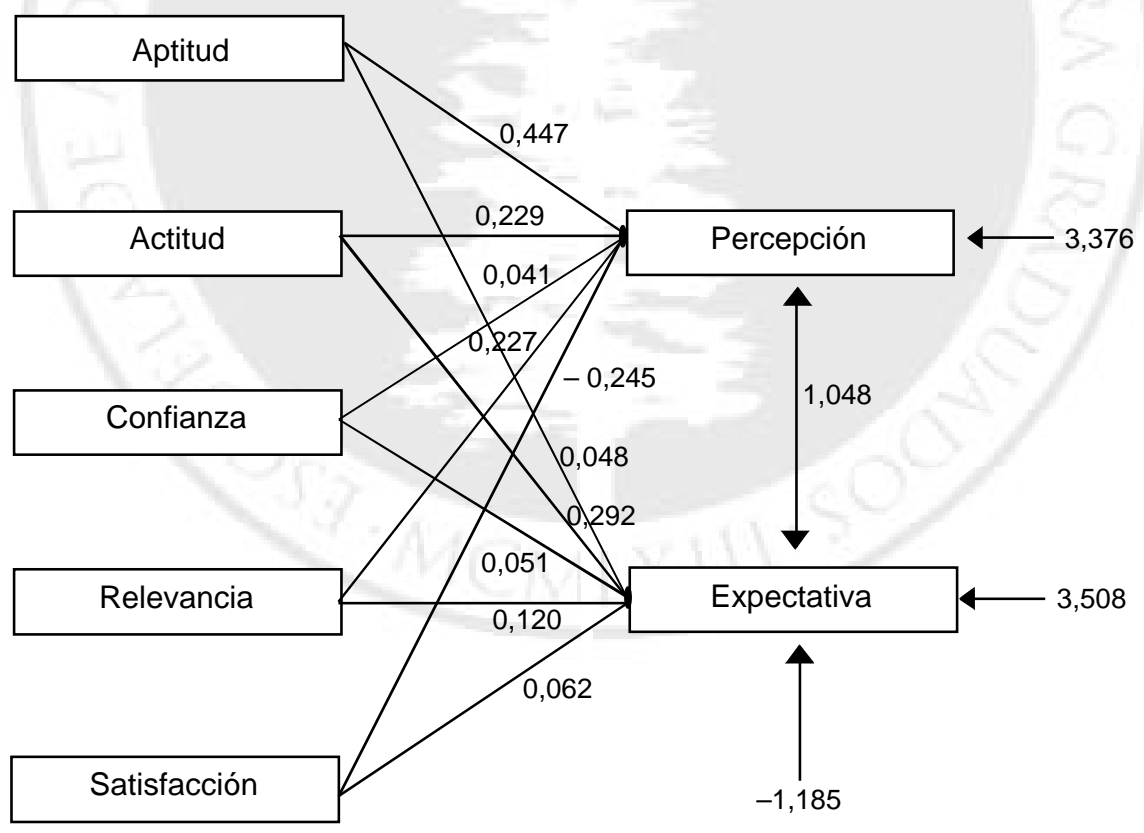

Modelo de evaluación de la percepción y la expectativa (Cardona, 2004) 


\subsection{Métodos}

Los coeficientes del modelo se calcularon usando conceptos de análisis multivariante, y se verificaron los supuestos básicos de normalidad para las variables métricas. Para las variables binomiales, se asumió su aproximación a la distribución normal debido al tamaño de la muestra. A fin de probar la existencia de los constructos propuestos en el modelo, se usó un análisis factorial por componentes principales como técnica de confirmación con rotación ortogonal. Se verificó que los constructos resultantes tenían altas comunalidades y explicaban un alto porcentaje de la varianza.

Para caracterizar la percepción y expectativa ciudadanas se usaron dos aproximaciones diferentes. La primera fue un análisis de cluster, mediante la aplicación de la metodología k-means, a fin de obtener las clases más homogéneas posibles. Cada una de estas clases se definió a partir de diferencias de medias entre grupos de las variables ilustrativas. La segunda aproximación se hizo usando un análisis de ANOVA para verificar las tendencias ciudadanas descritas en las hipótesis sobre la percepción y la expectativa. Se crearon intervalos de confianza para las medias de todas las variables relevantes y los constructos hallados.

\subsection{Datos}

Desde la perspectiva de la eficiencia y después de una cuidadosa revisión teórica y evaluación práctica entre un amplio rango de metodologías existentes, el método de recolección seleccionado fue un sistema mixto: entrevista telefónica complementada con entrevista electrónica. Esta decisión no afectó la calidad de las respuestas y mejoró el porcentaje de participación.

Para tomar la muestra de ciudadanos y previa consulta con expertos, el universo del estudio se definió como aquellos ciudadanos con la necesidad de interactuar con la administración pública para desarrollar trámites usando las TIC. En el caso peruano, se aplicó un análisis de outliers a fin de corregir los posibles errores, también se verificó que hubiera suficiente información, porque la relación entre el número de casos (463) y el número de constructos resultantes (7) era mayor que 1:20 (Hair, et al., 1999). La prueba de esfericidad de Barlett aseguró la ausencia de correlaciones. Usando la matriz de correlaciones parciales como matriz de antiimágenes, se demostró que existían altas intercorrelaciones. Aplicando la medida de adecuación de la muestra $(0,707)$ y de acuerdo con el índice de KaiserMeyer-Olkin, todas las variables usadas en el caso peruano eran buenas o muy buenas, con un Cronbach's Alpha $=0,7326$ (Brown, 2002; Hair et al., 1999). Los resultados específicos se pueden consultar en http://dsi.esade.edu/ dcardona/tesis (20 de diciembre, 2004).

\section{Conclusiones}

En cuanto a la percepción, usando los intervalos de confianza de cada variable existe suficiente evidencia estadística para concluir que la población peruana representada por la muestra considera que:

- Hay mayor confianza con los servicios electrónicos que con los servicios prestados tradicionalmente. 
- Los servicios electrónicos prestados por la administración pública son relevantes.

- Existe un mayor grado de satisfacción con los servicios electrónicos que con los servicios tradicionales.

En cuanto a la expectativa, usando los datos obtenidos es posible concluir que la muestra de la población peruana entrevistada considera que:

- El sistema más deseado de interacción con la administración pública es vía página web, e-mail o Internet.

- El proceso más deseado es la realización de trámites y el intercambio de información.
- El área más deseada para interactuar es la de empleo.

- El beneficio más deseado es la reducción de costos.

De manera general, existe suficiente evidencia estadística para concluir que la población peruana representada por la muestra considera que las TIC impactan su percepción de que los servicios ofrecidos por la administración pública vía Internet han mejorado su relación con ella. En contraste, no existe suficiente evidencia estadística para concluir que la población peruana representada por la muestra considera que en caso de interactuar con la administración pública vía Internet esperaría una excelente relación.

\section{Referencias bibliográficas}

BERMÚDEZ, P. (pablober@hotmail.com). 2004. Datos Perú. E-mail a D. Cardona (d.cardona.m@esade.edu).

BROWN, J. 2002. The Cronbach Alpha reliability estimate. Shiken: JALT Testing \& Evaluation SIG Newsletter. Vol 6, n. ${ }^{\circ}$, págs. 12-15.

BUSTAMANTE, A. 2002. La sociedad de la información en Perú: presente y perspectivas, 2002-2005. Lima: Telefónica del Perú.

CARDONA, D. 2004. Las tecnologías de la información y las comunicaciones (TIC) en la relación administración pública-ciudadano: la declaración electrónica de impuestos en una evaluación comparativa del caso colombiano y peruano. Tesis para optar el grado de Ph.D. in Management Sciences. ESADE-Universidad Ramón Llull, Barcelona, España.

COMPEAU, D.; HIGGINS, C., y HUFF, S. 1999. Social cognitive theory and individual reactions to computing technology: A longitudinal study. MIS Quarterly. Vol 23, n. ${ }^{\circ}$ 2, págs. 145-158.

DAVIS, F. 1989. Perceived usefulness, perceived ease of use, and user acceptance of information technology. MIS Quarterly. Vol. 13, n. ${ }^{\circ}$ 3, págs. 318-340.

DAVIS, F.; BAGOZZI, R. y WARSHAW, P. 1989. User acceptance of computer technology: A comparison of two theoretical models. Management Science. Vol. 35, n. ${ }^{\circ}$, págs. 982-1003. 
DAVIS, F. y VENAKATESH, V. 1996. A model of the antecedents of perceived ease of use: Development and test. Decision Sciences. Vol. 27, n. ${ }^{\circ}$, págs. 451-481.

2000. A theoretical extension of the technology acceptance model: Four longitudinal fields studies. Managemenet Science. Vol. 46, n. ${ }^{\circ}$ 2, págs. 186-204.

2004. Toward preprototype user acceptance testing of new information systems: Implications for software project management. IEEE Transactions on Engineering Management. Vol. 51, n. ${ }^{\circ}$, págs. 31-47.

DUTTA, S., y LOPEZ-CLAROS, A. 2005. Global information technology readiness report 2004-2005. Geneva, Switzerland: World Economic Forum.

FISHBEIN, M. y AJZEN, I. 1980. Understanding attitudes and predicting social behaivor. New Jersey: Prentice Hall.

GEFEN, D. y STRAUB, D. W. 1997. Gender differences in the perception and use of e-mail: An extension to the technology acceptance model. MIS Quarterly. Vol. 21, n. ${ }^{\circ}$, págs. $389-400$.

GUALTIERI, R. 1999. Impact of the emerging information society on the policy development process and democratic quality. París: OECD.

HAIR, J.; ANDERSON, R.; TATHAM, R. y BLACK, W. 1999. Análisis multivariante. D. Cano (trad.), 5 ed. Madrid: Prentice Hall Iberia.

HAN, S. 2003. Individual adoption of information systems in organisations: A literature review of technology acceptance model. Turku, Finland: Institute for Advanced Management Systems Research (IAMSR).

HENERSON, M.; Morris, L. L. y FITZ-GIBBON, C. T. 1987. How to measure attitudes. 2 ed. London: Sage.

MATHIESON, K. 1991. Predicting user intentions: Comparing the technology acceptance model with the theory of planned behavior. Information Systems Research. Vol. 2, n. ${ }^{\circ}$, págs. 173-191.

OECD. 2000 Understanding the digital divide. http://www.oecd.org/dsti/sti/prod/ Digital_divide.pdf [2002].

PARRA, R. (rparra@pcm.gob.pe). 2004. Los datos de e-gov en Perú. E-mail a D. Cardona (d.cardona.m@esade.edu).

PASCO, J. C. 2005. Análisis de la estrategia de gobierno electrónico en Perú. Lima: Governa.

ROGERS, E. M. 1995. Diffusion of innovations. 4 ed. New York: Free Press.

RUBIO, L. 2000. La percepción del ciudadano en la mejora de la calidad de los servicios públicos. En Longo, F. y Zafra, M. (ed.). Pensar lo público. Barcelona: Unión Iberoamericana de Municipalistas, ESADE. Págs. 353-379.

SNI. 2002. Plan Nacional de Informática. http://www.pcm.gob.pe/portal_ongei/publica/sni/pni.pdf [2004].

TAYLOR, S. y TODD, P. A. 1995. Understanding information technology usage: A test of competing models. Information Systems Research. Vol. 6, n. ${ }^{\circ}$ 2, págs. 144176. 